\section{Prevalência de hipertensão arterial em comunidades ribeirinhas do Rio Madeira, Amazônia Ocidental Brasileira}

\author{
Prevalence of arterial hypertension in communities \\ along the Madeira River, Western Brazilian \\ Amazon
}

\author{
La prevalencia de la hipertensión en las \\ comunidades que bordean el río Madeira, \\ occidente de la Amazonia brasileña
}

\begin{abstract}
The aim of this cross-sectional study was to estimate the prevalence of hypertension among adults $(n=841)$ in communities along the Madeira River in the Brazilian Amazon, prior to startup of the Santo Antônio Hydroelectric Plant. The study gathered information on sociodemographic conditions, history of diseases, habits, fish consumption, and anthropometric parameters. Logistic regression was used to calculate odds ratios and the respective confidence intervals. Among the riverine communities, 26\% (95\%CI: 23\%-29\%) of adults presented hypertension (29\% in men [95\%CI: 24\%-33\%] and 23\% in women [95\%CI: 19\%-27\%]). Factors associated with hypertension were age, BMI, and place of residence in men and age, triglycerides, and blood glucose in women. The findings can contribute to strategies for state and municipal health services to monitor and prevent cardiovascular events.
\end{abstract}

Hypertension; Amazonian Ecosystem; Prevalence
Beatriz Fátima Alves de Oliveira ${ }^{1}$

Dennys de Souza Mourão ${ }^{1}$

Núbia Gomes 2

Janaina Mara C. Costa ${ }^{2}$

Andreia Vasconcelos de Souza 2

Wanderley Rodrigues Bastos 2

Marlon de Freitas Fonseca 3

Carolina Fiorillo Mariani 4

Guilherme Abbad 4

Sandra S. Hacon 1

\section{Resumo}

O objetivo deste estudo foi estimar a prevalência de hipertensão em adultos residentes nas comunidades ribeirinhas do rio Madeira, previamente à operação da Usina Hidrelétrica Santo Antônio. Estudo transversal da prevalência de hipertensão arterial realizado com 841 adultos. Informações sobre condições sociodemográficas, morbidade referida, hábitos de vida, consumo de peixe e dados antropométricos foram avaliados. O método de regressão logística foi utilizado para estimar $a$ odds ratio e seus respectivos intervalos de confiança. Entre os ribeirinhos, 26\% (IC95\%: 23\%29\%) dos adultos apresentaram hipertensão: 29\% entre os homens (IC95\%: 24\%-33\%) e 23\% entre as mulheres (IC95\%: 19\%-27\%). Nos homens, os fatores associados à hipertensão foram idade, IMC, glicemia e local de residência. Entre as mulheres, os fatores preditivos foram idade triglicerídeos e glicemia. Os resultados deste estudo podem contribuir com a formulação de estratégias de monitoramento para prevenção de eventos cardiovasculares por parte dos órgãos de saúde estadual e municipal.

Hipertensão; Ecossistema Amazônico; Prevalência 


\section{Introdução}

A hipertensão arterial sistêmica (HAS) é um dos mais frequentes fatores de risco para doenças cardiovasculares ${ }^{1}$. No ano 2000 , o número estimado de adultos no mundo com hipertensão foi de 972 milhões, com previsão de $60 \%$ de aumento na prevalência dessa doença para o ano 2025 2. No Brasil, um estudo de revisão sobre a HAS mostra prevalência em torno de $20 \%$, com disparidades entre as regiões do país 3 . Dados de 2010 do Sistema de Vigilância de Fatores de Risco e Proteção para Doenças Crônicas por Inquérito Telefônico (VIGITEL) mostraram que a frequência de diagnóstico médico da HAS variou de $18 \%$ na Região Norte e $25 \%$ na Região Sudeste 4 .

A HAS é uma condição que favorece a aterogênese e o consequente desenvolvimento de processos isquêmicos cardíaco, cerebral, vascular periférico e renal, os quais também estão associados ao consumo de alimentos industrializados, sedentarismo e obesidade 1,5,6,7,8,9. A vigilância de fatores como a obesidade e o sedentarismo tem sido uma das formas mais efetivas para estabelecer medidas de prevenção primária e detecção precoce de doenças cardiovasculares ${ }^{10}$. No entanto, a adoção de tais medidas ainda constitui um desafio para gestores de saúde, especialmente em áreas isoladas como populações quilombolas, indígenas e ribeirinhas.

Na Amazônia Ocidental, as populações tradicionais ribeirinhas se caracterizam por desenvolverem atividades de subsistência (como o extrativismo, a agricultura familiar e a atividade pesqueira) e vêm apresentando mudanças em seus hábitos de vida em virtude de recentes projetos de desenvolvimento, particularmente na área de infraestrutura. Em relação às comunidades localizadas às margens do rio Madeira, destacamse as obras de construção da barragem de Santo Antônio e Jirau, que estão localizadas próximas à cidade de Porto Velho, Rondônia, Brasil. Os impactos socioambientais oriundos desses empreendimentos sobre o estilo de vida podem ser suficientemente importantes a ponto de alterar a qualidade de vida e o perfil de morbimortalidade da população ribeirinha. Uma preocupação seria a possibilidade de aumento da prevalência de HAS na população residente em áreas de influência (direta e indireta) desses empreendimentos.

Estudos prévios sobre a situação de saúde da população ribeirinha e seus principais condicionantes permitirão comparações com avaliações futuras e a possível detecção das fragilidades do sistema, além de ampliar o entendimento da magnitude e as tendências históricas dos riscos de morbidade e mortalidade nesses grupos. Nesse contexto, este estudo objetiva estimar a preva- lência de HAS em adultos residentes nas comunidades ribeirinhas previamente ao represamento do rio Madeira para o funcionamento da Usina Hidrelétrica Santo Antônio.

\section{Materiais e métodos}

\section{Desenho do estudo}

Estudo transversal, observacional e de caráter descritivo da prevalência da HAS na população adulta (idade superior a 16 anos) residente às margens do rio Madeira, Município de Porto Velho, no período de 2009-2011. Foram estudadas 15 comunidades ribeirinhas, 14 delas localizadas próximas à cachoeira de Santo Antônio, local de construção da Usina Hidrelétrica Santo Antônio e outra, a Reserva de Cuniã, situada a $180 \mathrm{~km}$ da cidade de Porto Velho. A Reserva de Cuniã foi incluída como uma área de referência neste estudo por causa dos típicos hábitos alimentares de sua população e do seu isolamento geográfico.

Embora a proposta inicial fosse uma pesquisa por amostragem, as necessidades identificadas durante o desenvolvimento do trabalho demandaram a realização de um censo. As demandas foram atribuídas a motivos administrativos, acesso aos domicílios e para evitar maiores conflitos com algumas das comunidades que naquele momento estavam passando por processo de reassentamento e indenização de seus domicílios. Do total de 1.188 adultos convidados a participar do estudo, 841 (70\%) aderiram ao estudo de hipertensão, sendo 701 de comunidades ribeirinhas do rio Madeira e 140 da Reserva de Cuniã.

Ao longo do trabalho, respeitando os direitos e a vontade dos indivíduos, foram inevitáveis as perdas nas etapas do projeto em relação à aferição da pressão arterial, à avaliação antropométrica e à coleta de sangue. De 841 participantes que aderiram ao estudo, 631 (75\%) foram submetidos à avaliação antropométrica (peso e altura) e 539 (65\%) tiveram amostra de sangue analisada para determinação da concentração de glicose e triglicerídeos no plasma. A distribuição das perdas do estudo segundo faixa etária e sexo foi avaliada para identificar potenciais distorções nos resultados encontrados.

A pressão arterial foi aferida duas vezes em conformidade com as recomendações das $V D i$ retrizes Brasileiras de Pressão Arterial 7 - técnica auscultatória e esfigmomanômetro aneróide previamente calibrado (Becton, Dickinson and Company, Franklin Lakes, Estados Unidos). Foi definido como portador de HAS o indivíduo que apresentou pressão arterial sistólica maior 
ou igual 140mmHg e diastólica maior ou igual a 90mmHg ou que estava fazendo uso diário e/ou regular de medicação para controle da HAS no mínimo 6 meses antes da realização do estudo.

Um questionário semiestruturado com base em questionários validados pelo Ministério da Saúde foi aplicado a todos os participantes. O questionário incluiu módulos específicos sobre as condições sociodemográficas, morbidade referida, hábitos de vida (tabagismo, etilismo) e inquérito alimentar com detalhamento do consumo semanal de peixe. Os entrevistadores foram submetidos a treinamento prévio para aplicação dos questionários e supervisionados pela coordenação de campo. As seguintes variáveis foram consideradas: sexo (masculino/feminino); idade (16-29 anos, 30-39 anos, 40-49 anos, 50-59 anos, 60 anos ou mais); tabagismo (sim/não), etilismo (nenhuma, 1 ou mais vezes por semana); consumo de peixe (mais de 3 vezes na semana, menos de 3 vezes na semana); escolaridade (até 2 anos de estudo, 6-9 anos de estudo, 10 ou mais anos de estudo); renda mensal da família (menos de 1 salário, 1-3 salários, mais de 3 salários - valor de referência para um salário mínimo: $\mathrm{R} \$ 512,00$ ).

As referências geográficas (espaciais) utilizadas para agrupamento das comunidades foram suas relações com a margem do rio Madeira (direita ou esquerda em relação ao fluxo das águas) e suas posições (a montante ou a jusante) em relação à área de construção da Usina Hidrelétrica Santo Antônio (cachoeira de Santo Antônio). Admite-se que, em geral, as comunidades localizadas na margem esquerda possuam menor contato com a área urbana e que, em relação à cachoeira, as comunidades situadas à montante caracterizam áreas de influência direta na represa (em virtude do alagamento necessário ao funcionamento da hidrelétrica) enquanto algumas comunidades localizadas à jusante da hidrelétrica estão em áreas de influência indireta. Embora localizada à jusante de Porto Velho, a Reserva de Cuniã foi tratada como uma área de referência neste estudo, não tendo sido considerada uma área de influência da hidrelétrica.

Para as coletas dos dados antropométricos, foram utilizados balança digital (Balança Digital G-TECH Glass PRO devidamente testada e calibrada; Glicomed, Rio de Janeiro, Brasil) e um estadiômetro portátil de coluna de madeira e alumínio anodizado. Para o cálculo do índice de massa coporal (IMC), usou-se o peso do indivíduo (em quilogramas), dividido pela sua altura (em metros) ao quadrado. Os valores de IMC foram categorizados em baixo peso/normal (IMC < 25kg.m-2) ou em excesso de peso (IMC $\geq$ $25 \mathrm{~kg} \cdot \mathrm{m}^{-2}$ ), categoria esta que inclui sobrepeso e obesidade 11 .
As coletas de sangue foram de responsabilidade do laboratório do Hospital 9 de Julho CEACLIN, localizado no Município de Porto Velho. As amostras foram coletadas com seringa de $10 \mathrm{~mL}$ com agulha $25 \mathrm{x} 7 \mathrm{BD}$ descartáveis, e transferidas para os respectivos tubos de coleta (vacutainer) com fluoreto de sódio para dosagem de glicose e sem anticoagulante com gel separador para demais ensaios imunológicos. O método colorimétrico enzimático foi usado para determinação das concentrações plasmáticas de glicose e de triglicerídeos (equipamento Roche Hitachi 911, Chemistry Analyzer Spectrophotometer, Nova York, Estados Unidos; kits Labtest, Health Testing Centers, Saint Louis, Estados Unidos), que foram dicotomizadas como valores normais ou elevados. As concentrações de glicose e triglicerídeos foram consideradas elevadas quando superiores a $100 \mathrm{mg} \%$ e $150 \mathrm{mg} \%$, respectivamente.

$\mathrm{Na}$ análise estatística, foi realizada análise exploratória dos dados com a distribuição percentual das variáveis indicativas de condições demográficas (idade e sexo), localização das residências (seção do rio, margem), IMC, hábitos de vida (tabagismo e etilismo) e hábitos alimentares (consumo de peixe e fritura). A prevalência de HAS foi calculada com seus respectivos intervalos de 95\% de confiança (IC95\%), segundo sexo. O teste qui-quadrado $\left(\chi^{2}\right)$ foi aplicado para verificar as diferenças da prevalência de HAS entre homens e mulheres ao nível de 5\% de significância. $\mathrm{O}$ teste ainda foi usado para averiguar se aqueles que participaram da avaliação antropométrica e exames laboratoriais tinham as mesmas características demográficas quando comparados àqueles que não participaram.

Para estimar a associação entre as variáveis preditoras e a variável dependente (hipertensão arterial), o modelo de regressão logística foi utilizado para obtenção das estimativas de razões de chance (odds ratio - OR) e seus IC95\%. Na análise bivariada, as variáveis que apresentaram associação com HAS ao nível de $20 \%$ foram incluídas na análise multivariada. Foram mantidas no modelo ajustado as variáveis que apresentaram nível de significância estatística (valor de $\mathrm{p}<0,05$ ). O ajuste do modelo foi avaliado pelo critério de Akaike (AIC - Akaike Information Criteria) e o método ANOVA. O banco de dados foi organizado no programa Microsoft Access (Microsoft Corp., Estados Unidos) e todas as análises estatísticas foram realizadas com suporte do programa Microsoft Excel (Microsoft Corp., Estados Unidos) e Linguagem R (The R Foundation for Statistical Computing, Viena, Áustria; http:// www.r-project.org). 
Este trabalho é parte integrante do estudo de Avaliação de Impacto à saúde em comunidades potencialmente expostas a mercúrio no rio Madeira, apoiado pelos projetos INOVA ENSP e Universal/CNPq (processo oo 478085/2010-9), e fo desenvolvido em parceria com as seguintes instituições: Escola Nacional de Saúde Pública Sergio Arouca (ENSP/Fiocruz), Instituto Nacional da Saúde da Mulher da Criança e do Adolescente Fernandes Figueira (IFF/Fiocruz), Universidade Federal de Rondônia (UNIR), Universidade Federal do Rio de Janeiro (UFRJ) e Pontifícia Universidade Católica do Rio de Janeiro (PUC-RJ). Este estudo foi realizado no âmbito do Programa de Monitoramento Hidrobiogeoquímico da Santo Antônio Energia S/A, empresa responsável pela construção e operação da Usina Hidrelétrica Santo Antônio, em Porto Velho. A pesquisa foi aprovada pelo Comitê de Ética da ENSP e pelo Comitê de Ética da UNIR, considerando todos os aspectos éticos da Resolução no 196/1996 do Conselho Nacional de Saúde.

\section{Área e população de estudo}

A cidade de Porto Velho possui uma área territorial de $34.068,50 \mathrm{~km}^{2}$ e concentra aproximadamente $30 \%$ da população do estado. Localizada na Amazônia Ocidental, seu centro urbano fica à margem direita do rio Madeira (um dos mais importantes afluentes do rio Amazonas). A cachoeira de Santo Antônio (onde está sendo construída a Usina Hidrelétrica Santo Antônio) está localizada a 7 quilomêtros acima da cidade de Porto Velho.

Cerca de $91 \%$ da população de Porto Velho concentram-se na área urbana. O Índice de Desenvolvimento Humano (IDH) dessa cidade é de 0,763 . Em termos de mobilidade espacial, a população ribeirinha é considerada mais estável quando comparada com a população urbana e reside em núcleos comunitários constituídos, em média, de 20-30 domicílios dispersos às margens dos rios e igarapés da região, sem endereço. As fontes de renda da população ribeirinha derivam prioritariamente da pesca.

Além da população ribeirinha periurbana do Madeira, o estudo incluiu também os 5 núcleos da Reserva de Cuniã, localizada à margem esquerda do baixo Madeira, cerca de $180 \mathrm{~km}$ da cidade de Porto Velho. A Reserva de Cuniã possui uma área territorial de 55.850ha com duas áreas distintas: terra firme e várzea. A última é regulada pela sazonalidade da região, com estiagem (maio a outubro) e cheia (novembro a abril). Essa reserva é formada por aproximadamente 400 pessoas, incluindo adultos e crianças, e possui características sociodemográficas e ambientais peculiares que a torna diferenciada quando comparada às demais comunidades ribeirinhas do rio Madeira incluídas neste estudo. É uma comunidade tradicional de pescadores que depende quase integralmente dos recursos pesqueiros da região. $\mathrm{O}$ isolamento geográfico é importante, particularmente, em razão das dificuldades de acesso por barco, no período de seca.

Todas as comunidades ribeirinhas consideradas neste estudo estão localizadas às margens do rio Madeira, incluindo aquelas localizadas em áreas urbanas de Porto Velho (Figura 1).

\section{Resultados}

Do total de 841 adultos, predominaram indivíduos com idade entre 16 a 29 anos (30\%), sobrepesos/obesidade (55\%), ribeirinhos residentes da margem esquerda (52\%) e à montante da Usina Hidrelétrica de Santo Antônio (46\%). Em geral, observou-se maior distribuição percentual de fumantes $(55 \%)$, nenhum consumo de bebida alcoólica (64\%), ingestão de peixe até três vezes na semana (52\%). Aproximadamente $70 \%$ dos adultos possuem de 3 a 9 anos de estudo e $36 \%$ referem renda mensal de 1 a 3 salários mínimos (Tabela 1).

Em relação ao sexo, verificaram-se diferenças percentuais estatisticamente significantes entre homens e mulheres para as seguintes variáveis: faixa etária, IMC, margem do rio, etilismo e escolaridade (valor de $\mathrm{p}<0,05$ ). Entre as mulheres, $62 \%$ apresentaram sobrepeso e $32 \%$ possuem entre 16 a 29 anos. Do total de homens, $71 \%$ possuem de 3 a 6 anos de estudo, $63 \%$ são fumantes e $48 \%$ consomem bebida alcoólica pelo menos 1 vez na semana (Tabela 1 ).

A prevalência de HAS foi de 25,7 (IC95\%: 22,728,6), com maior predominância no sexo masculino (28,7; IC95\%: 24,3-33,0) quando comparada à prevalência no sexo feminino (22,8; IC95\%: 18,8-26,7). A prevalência de HAS apresentou tendência crescente ao longo das faixas etárias, com maior valor entre indivíduos com mais de 60 anos (58,6; IC95\%: 49,6-67,4). A população ribeirinha que reside na seção à jusante da Usina Hidrelétrica Santo Antônio apresentou maior prevalência de HAS (34,5; IC95\%: 29,2-39,8) quando comparada à prevalência da seção à montante $\mathrm{e}$ Reserva de Cuniã (valor de $\mathrm{p}<0,05$ ). Ainda, indivíduos com excesso de peso e níveis elevados de triglicerídeos e de glicemia apresentaram maior prevalência de HAS quando comparados, respectivamente, aos indivíduos com peso adequado, triglicerídeos e de glicemia dentro dos padrões de normalidade (Tabela 2 ).

As razões de prevalência de HAS entre homens e mulheres foram significativas nas seguin- 


\section{Figura 1}

Localização geográfica das comunidades ribeirinhas do Rio Madeira. Porto Velho, Rondônia, Brasil, 2010-2011.
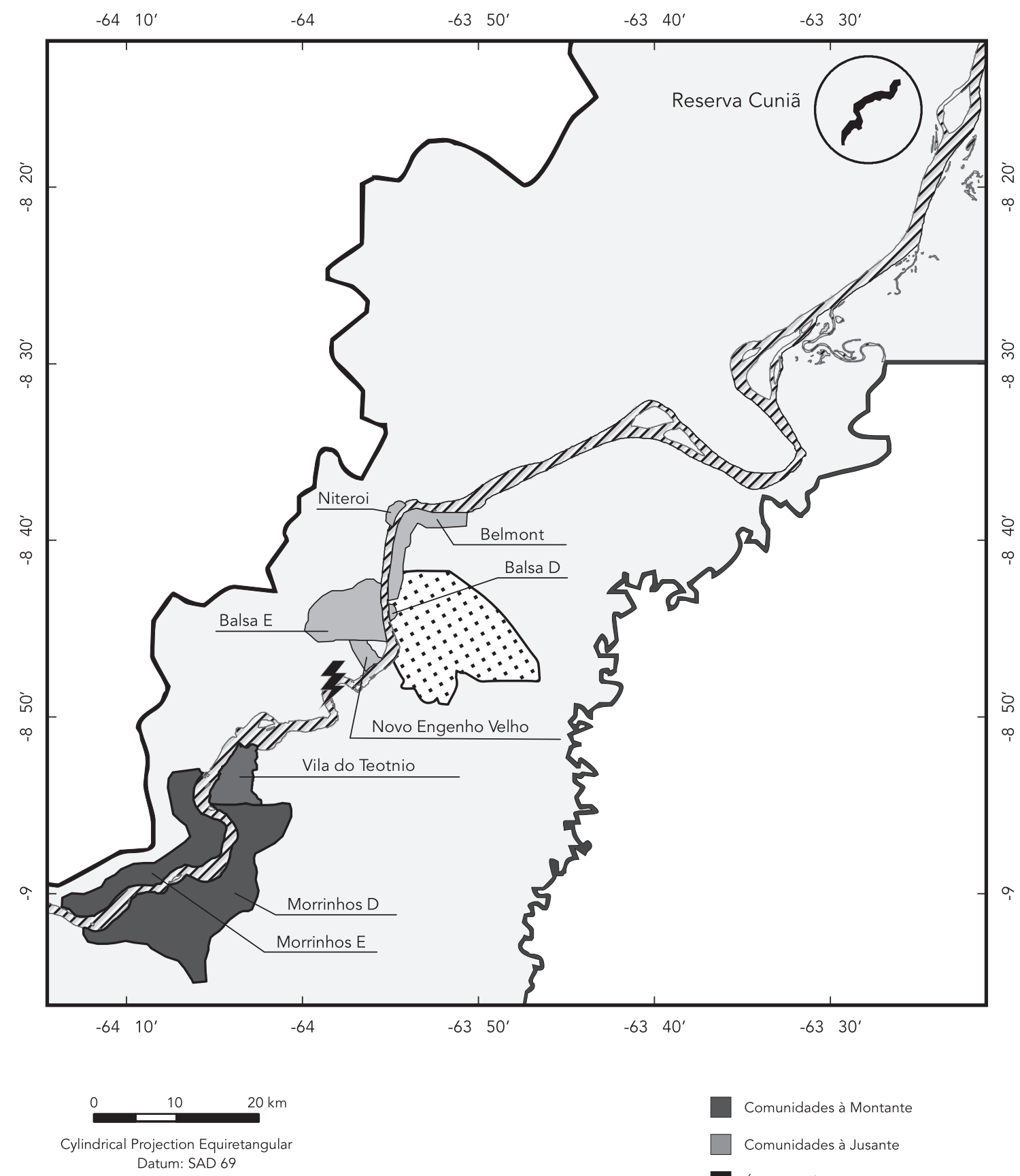

Comunidades à Montante

Comunidades à Jusante

Área de referência (Reserva de Cuniã)

3 Usina Hidrelétrica Santo Antônio

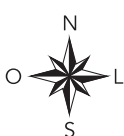

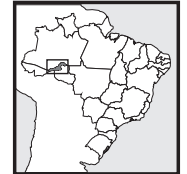

Município de Porto Velho

Rio Madeira

Área urbana

Fluxo do rio 
Tabela 1

Distribuição percentual da população residente em áreas ribeirinhas, segundo sexo. Porto Velho, Rondônia, Brasil, 2011.

\begin{tabular}{|c|c|c|c|c|c|c|c|}
\hline & \multicolumn{2}{|c|}{ Masculino } & \multicolumn{2}{|c|}{ Feminino } & \multicolumn{2}{|c|}{ Total } & \multirow[t]{2}{*}{ Valor de $p$ * } \\
\hline & $\mathrm{n}$ & $\%$ & $n$ & $\%$ & $\mathrm{n}$ & $\%$ & \\
\hline \multicolumn{8}{|l|}{ Idade (anos) } \\
\hline $16-29$ & 103 & 25,3 & 140 & 32,3 & 243 & 28,9 & 0,000 \\
\hline $30-39$ & 85 & 20,9 & 124 & 28,6 & 209 & 24,9 & \\
\hline $40-49$ & 80 & 19,7 & 76 & 17,5 & 156 & 18,5 & \\
\hline $50-59$ & 69 & 17,0 & 46 & 10,6 & 115 & 13,7 & \\
\hline 60 ou mais & 70 & 17,2 & 48 & 11,1 & 118 & 14,0 & \\
\hline Total & 407 & 100,0 & 434 & 100,0 & 841 & 100,0 & \\
\hline \multicolumn{8}{|l|}{ IMC ** } \\
\hline Baixo peso/Normal & 159 & 53,2 & 125 & 37,7 & 284 & 45,0 & 0,000 \\
\hline Excesso de peso & 140 & 46,8 & 207 & 62,3 & 347 & 55,0 & \\
\hline Total & 299 & 100,0 & 332 & 100,0 & 631 & 100,0 & \\
\hline \multicolumn{8}{|l|}{ Margem do rio } \\
\hline Direita & 181 & 44,5 & 224 & 51,6 & 405 & 48,2 & 0,038 \\
\hline Esquerda & 226 & 55,5 & 210 & 48,4 & 436 & 51,8 & \\
\hline Total & 407 & 100,0 & 434 & 100,0 & 841 & 100,0 & \\
\hline \multicolumn{8}{|c|}{ Localização das comunidades } \\
\hline Jusante & 144 & 35,4 & 166 & 38,2 & 310 & 36,9 & 0,654 \\
\hline Montante & 192 & 47,2 & 199 & 45,9 & 391 & 46,5 & \\
\hline Reserva de Cuniã & 71 & 17,4 & 69 & 15,6 & 140 & 16,6 & \\
\hline Total & 407 & 100,0 & 434 & 100,0 & 841 & 841 & \\
\hline \multicolumn{8}{|l|}{ Tabagismo } \\
\hline Não & 152 & 37,3 & 229 & 52,8 & 381 & 45,3 & 0,000 \\
\hline Sim & 255 & 62,7 & 205 & 47,2 & 460 & 54,7 & \\
\hline Total & 407 & 100,0 & 434 & 100,0 & 841 & 100,0 & \\
\hline \multicolumn{8}{|c|}{ Etilismo (vezes por semana) } \\
\hline Nenhuma & 212 & 52,1 & 330 & 76,0 & 542 & 64,4 & 0,000 \\
\hline 1 ou mais & 195 & 47,9 & 104 & 24,0 & 299 & 35,6 & \\
\hline Total & 407 & 100,0 & 434 & 100,0 & 841 & 100,0 & \\
\hline \multicolumn{8}{|c|}{ Consumo de peixe (vezes por semana) } \\
\hline Mais de 3 & 197 & 48,9 & 203 & 47,3 & 410 & 48,1 & 0,652 \\
\hline Até 3 & 206 & 51,1 & 226 & 52,7 & 432 & 51,9 & \\
\hline Total & 403 & 100,0 & 429 & 100,0 & 832 & 100,0 & \\
\hline \multicolumn{8}{|l|}{ 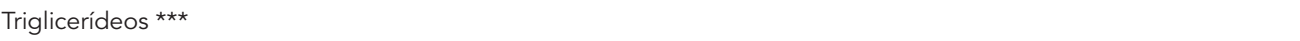 } \\
\hline Normal & 217 & 82,5 & 210 & 76,4 & 427 & 79,4 & 0,078 \\
\hline Elevado & 46 & 17,5 & 65 & 23,6 & 111 & 20,6 & \\
\hline Total & 263 & 100,0 & 275 & 100,0 & 538 & 100,00 & \\
\hline \multicolumn{8}{|l|}{ Glicemia \# } \\
\hline Normal & 204 & 77,3 & 227 & 82,5 & 431 & 80,0 & 0,126 \\
\hline Elevada & 60 & 22,7 & 48 & 17,5 & 108 & 20,0 & \\
\hline Total & 264 & 100,0 & 275 & 100,0 & 539 & 100,0 & \\
\hline \multicolumn{8}{|c|}{ Escolaridade (anos de estudo) } \\
\hline Mais de 10 & 65 & 16,2 & 88 & 20,5 & 153 & 18,4 & 0,204 \\
\hline $3-9$ & 285 & 70,9 & 281 & 65,5 & 566 & 68,1 & \\
\hline Até 2 & 52 & 12,9 & 60 & 14,0 & 112 & 13,5 & \\
\hline Total & 402 & 100,0 & 429 & 100,0 & 831 & 100,0 & \\
\hline
\end{tabular}

(continua) 


\begin{tabular}{|c|c|c|c|c|c|c|c|}
\hline & \multicolumn{2}{|c|}{ Masculino } & \multicolumn{2}{|c|}{ Feminino } & \multicolumn{2}{|c|}{ Total } & \multirow[t]{2}{*}{ Valor de $p$ * } \\
\hline & $\mathbf{n}$ & $\%$ & $\mathbf{n}$ & $\%$ & $\mathrm{n}$ & $\%$ & \\
\hline \multicolumn{8}{|c|}{ Renda familiar (salários mínimo) \#\# } \\
\hline Mais de 3 & 116 & 34,5 & 120 & 33,7 & 236 & 34,1 & 0,960 \\
\hline $1-3$ & 123 & 36,6 & 130 & 36,5 & 253 & 36,6 & \\
\hline Até 1 & 97 & 28,9 & 106 & 29,8 & 203 & 29,3 & \\
\hline Total & 366 & 100,0 & 356 & 100,0 & 692 & 100,0 & \\
\hline
\end{tabular}

IMC: índice de massa corporal.

* Teste qui-quadrado para diferenças das categorias entre sexo.

** Baixo peso/normal: IMC $<25$ kg.m-2; excesso de peso: IMC $\geq 25 \mathrm{~kg} \cdot \mathrm{m}-2$;

*** Normal: triglicerídeos < 150mg\%; elevado: triglicerídeos $\geq 150 \mathrm{mg} \%$;

\# Normal: glicemia < 100mg\%; elevada: glicemia $\geq 100 \mathrm{mg} \%$;

\#\# Valor de referência para um salário mínimo: $\mathrm{R} \$ 512,00$.

Tabela 2

Prevalência de hipertensão (\%), estratificada por sexo de acordo com características sociodemográficas, hábitos de vida e local de residência da população ribeirinha. Porto Velho, Rondônia, Brasil, 2009-2011.

\begin{tabular}{|c|c|c|c|c|}
\hline & $\begin{array}{c}\text { Masculino } \\
\text { HAS (IC95\%) }\end{array}$ & $\begin{array}{l}\text { Feminino } \\
\text { HAS (IC95\%) }\end{array}$ & $\begin{array}{c}\text { Total } \\
\text { HAS (IC95\%) }\end{array}$ & $\mathrm{RP} M / \mathrm{F}$ \\
\hline Global & $28,7(24,3-33,0)$ & $22,8(18,8-26,7)$ & $25,7(22,7-28,6)$ & 1,25 * \\
\hline \multicolumn{5}{|l|}{ Idade (anos) } \\
\hline $16-29$ & $18,4(10,9-25,9)$ & $5,0(1,3-8,6)$ & $10,7(6,8-14,6)$ & 3,68 * \\
\hline $30-39$ & $17,6(9,5-25,7)$ & $16,9(10,3-23,5)$ & $17,2(12,0-22,3)$ & 1,04 \\
\hline $40-49$ & $22,5(13,3-31,6)$ & $27,6(17,5-37,6)$ & $25,0(18,2-31,8)$ & 0,80 \\
\hline $50-59$ & $36,2(24,8-47,5)$ & $45,7(31,3-60,0)$ & $40,0(31,0-49,0)$ & 0,79 \\
\hline 60 ou mais & $57,1(49,0-76,9)$ & $60,4(37,3-72,3)$ & $58,6(49,6-67,4)$ & 0,94 \\
\hline \multicolumn{5}{|l|}{ 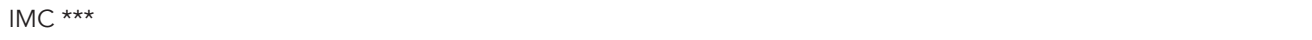 } \\
\hline Baixo peso/Normal & $23,9(17,3-30,5)$ & $21,6(14,4-28,8)$ & $22,9(18,0-27,8)$ & 1,10 \\
\hline Excesso de peso & $38,6(30,5-46,6)$ & $30,0(23,7-36,2)$ & $33,4(28,4-38,4)$ & 1,30 ** \\
\hline \multicolumn{5}{|l|}{ Margem } \\
\hline Direita & $31,5(24,7-38,2)$ & $23,2(17,7-28,7)$ & $26,9(22,6-31,2)$ & 1,36 ** \\
\hline Esquerda & $26,5(20,7-32,2)$ & $22,4(16,7-28,0)$ & $24,5(20,5-28,5)$ & 1,18 \\
\hline \multicolumn{5}{|l|}{ Localização } \\
\hline Jusante & $40,3(32,3-48,3)$ & $29,5(22,5-36,4)$ & $34,5(29,2-39,8)$ & 1,37 * \\
\hline Montante & $22,9(16,9-28,8)$ & $17,6(12,3-28,9)$ & $20,2(16,2-24,2)$ & 1,30 \\
\hline Reserva de Cuniã & $21,1(11,6-30,6)$ & $21,7(12,0-31,4)$ & $21,4(14,6-28,2)$ & 0,97 \\
\hline \multicolumn{5}{|l|}{ Tabagismo } \\
\hline Não & $29,6(22,3-36,8)$ & $18,8(13,7-23,8)$ & $23,1(18,9-27,3)$ & 1,57 * \\
\hline Sim & $28,2(22,8-33,7)$ & $27,3(21,2-33,4)$ & $27,8(23,7-32,0)$ & 1,03 \\
\hline \multicolumn{5}{|c|}{ Etilismo (vezes por semana) } \\
\hline Nenhuma & $27,4(21,4-33,4)$ & $24,8(20,1-29,4)$ & $25,8(22,1-29,5)$ & 1,10 \\
\hline 1 ou mais & $30,3(23,8-36,7)$ & $16,3(9,2-23,4)$ & $25,4(20,5-30,3)$ & 1,86 * \\
\hline \multicolumn{5}{|c|}{$\begin{array}{l}\text { Consumo de peixe (vezes por } \\
\text { semana) }\end{array}$} \\
\hline Mais de 3 & $25,9(19,8-32,0)$. & $24,6(18,7-30,5)$ & $25,3(21,0-29,6)$ & 1,05 \\
\hline Até 3 & $31,6(25,2-37,9)$ & $21,2(15,9-26,5)$ & $26,2(22,0-30,3)$ & 1,50 * \\
\hline
\end{tabular}

(continua) 


\begin{tabular}{|c|c|c|c|c|}
\hline & $\begin{array}{l}\text { Masculino } \\
\text { HAS (IC95\%) }\end{array}$ & $\begin{array}{c}\text { Feminino } \\
\text { HAS (IC95\%) }\end{array}$ & $\begin{array}{c}\text { Total } \\
\text { HAS (IC95\%) }\end{array}$ & RP M/F \\
\hline \multicolumn{5}{|l|}{ Triglicerídeos \# } \\
\hline Normal & $28,1(22,1-34,0)$ & $18,1(12,9-23,3)$ & $23,2(19,2-27,2)$ & 1,55 * \\
\hline Elevado & $37,0(23,0-51,0)$ & $46,2(34,0-58,3)$ & $42,3(33,1-51,5)$ & 0,80 \\
\hline \multicolumn{5}{|l|}{ Glicemia \#\# } \\
\hline Normal & $26,5(20,4-32,5)$ & $19,4(14,2-24,5)$ & $22,1(18,2-26,0)$ & 1,36 ** \\
\hline Elevada & $40,0(27,6-52,4)$ & $50,0(35,8-64,1)$ & $44,4(35,0-53,8)$ & 0,80 \\
\hline \multicolumn{5}{|c|}{ Escolaridade (anos) } \\
\hline Mais de 10 & $20,0(10,3-29,7)$ & $15,9(8,2-23,5)$ & $17,6(11,6-23,6)$ & 1,26 \\
\hline $3-9$ & $29,1(23,8-34,4)$ & $21,4(16,6-26,1)$ & $25,3(21,8-28,9)$ & 1,36 * \\
\hline Menos de 2 & $38,5(25,2-51,7)$ & $41,7(29,2-54,1)$ & $40,2(31,1-49,3)$ & 0,92 \\
\hline \multicolumn{5}{|c|}{ Renda (salários mínimos) \#\#\# } \\
\hline Mais de 3 & $23,3(15,6-31,0)$ & $19,2(12,1-26,2)$ & $21,2(16,0-26,4)$ & 1,21 \\
\hline $1-3$ & $33,3(25,0-41,6)$ & $28,5(20,7-36,2)$ & $30,8(25,1-36,5)$ & 1,17 \\
\hline Menos de 1 & $30,9(21,7-40,0)$ & $27,4(18,9-35,8)$ & $29,1(22,8-35,3)$ & 1,13 \\
\hline
\end{tabular}

HAS: hipertensão arterial sistêmica; IC95\%: intervalo de 95\% de confiança; IMC: índice de massa corporal; RP: razão de proporções.

$* p<0,05$

** $p<0,10$;

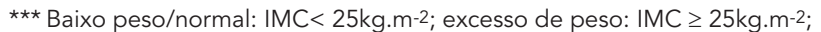

\# Normal: triglicerídeos < 150mg\%; elevado: triglicerídeos $\geq 150 \mathrm{mg} \%$;

\#\# Normal: glicemia < 100mg\%; elevada: glicemia $\geq 100 \mathrm{mg} \%$;

\#\#\# Valor de referência para um salário mínimo: $\mathrm{R} \$ 512,00$.

tes categorias: faixa etária de 16-29 anos; excesso de peso; residentes da margem direita; não fumantes; consumo de álcool (1 ou mais vezes na semana); consumo de peixe (até 3 vezes na semana); triglicerídeos e glicemia normais; e aqueles com 3-9 anos de estudo (Tabela 2).

A Tabela 3 exibe as estimativas de OR para HAS não ajustadas, estratificadas por sexo e de acordo com as características sociodemográficas, hábitos de vida e local de residência da população ribeirinha. Entre os homens, as variáveis que mostraram associação positiva com HAS foram idade maior que 50 anos, excesso de peso e residir em comunidades à jusante da cachoeira de Santo Antônio. Além disso, as estimativas de OR para HAS foram de 1,8 e 2,5 para homens com glicemia elevada e com menos de 2 anos de estudo comparados, respectivamente, aos homens com glicemia normal e com mais de 10 anos de estudo (valor de $\mathrm{p}<0,005$ ).

Para as mulheres, na análise bivariada todas as faixas etárias foram associadas positivamente com hipertensão quando comparadas às mulheres com idade entre 16 a 29 anos (valor de $\mathrm{p}<$ 0,001). Mulheres residentes na montante da Usina Hidrelétrica Santo Antônio apresentaram 50\% menor chance de ter HAS quando comparadas às mulheres residentes à jusante da usina (valor de $\mathrm{p}<0,05)$. A estimativa de OR para HAS foi de 1,6 para mulheres fumantes quando comparadas às mulheres não fumantes e de 3,9 para mulheres com triglicerídeos elevados quando comparadas àquelas com níveis normais de triglicerídeos. Em relação à glicemia, a chance de apresentar HAS foi maior entre as mulheres com glicemia elevada quando comparadas às mulheres com níveis normais de glicemia $\left(\mathrm{OR}_{\text {bruta }}=4,1\right.$; IC95\%: 2,2 8,0; valor de $\mathrm{p}<0,000$ ).

$\mathrm{Na}$ análise ajustada (Tabela 4), permaneceram associados à ocorrência de HAS em homens: idade superior a 60 anos ou mais $\left(\mathrm{OR}_{\text {ajustada }}=5,2\right.$; IC95\%: 2,3-12,0), excesso de peso $\left(\mathrm{OR}_{\text {ajustada }}=2,6\right.$; IC95\%: 1,4-4,6) e glicemia elevada $\left(\mathrm{OR}_{\text {ajustada }}=1,8\right.$; IC95\%: 1,0-3,4). Homens residentes à montante $\left(\mathrm{OR}_{\text {ajustada }}=0,4\right.$; IC95\%: 0,2-0,7) e na Reserva de Cuniã $\left(\mathrm{OR}_{\text {ajustada }}=0,4\right.$; IC 95\%: 0,2-0,8) apresentaram efeito protetor para HAS comparados aos homens que residem à jusante do local de construção da Usina Hidrelétrica Santo Antônio. Nas mulheres, a idade, triglicerídeos elevados e glicemia elevada mantiveram-se estatisticamente significante (valor de $\mathrm{p}<0,05$ ), após o ajuste com as outras variáveis. 
Tabela 3

Odds ratio não ajustada $\left(\mathrm{OR}_{\text {bruta }}\right)$ para hipertensão arterial sistêmica (HAS), estratificada por sexo de acordo com características spciodemográficas, hábitos de vida e local de residência da população ribeirinha. Porto Velho, Rondônia, Brasil, 2009-2011.

\begin{tabular}{|c|c|c|c|c|}
\hline & \multirow{2}{*}{$\begin{array}{c}\text { Masculino } \\
\text { OR }_{\text {bruta }}(\text { IC } 95 \%)\end{array}$} & \multicolumn{3}{|c|}{ Feminino } \\
\hline & & Valor de $\mathrm{p}$ & OR $_{\text {bruta }}(\mathrm{IC} 95 \%)$ & Valor de $p$ \\
\hline \multicolumn{5}{|l|}{ Idade (anos) } \\
\hline $16-29$ & 1,0 & & 1,0 & \\
\hline $30-39$ & $0,9(0,4-2,0)$ & 0,887 & $3,9(1,6-9,5)$ & 0,003 \\
\hline $40-49$ & $1,3(0,6-2,6)$ & 0,498 & $7,2(2,9-18,0)$ & 0,000 \\
\hline $50-59$ & $2,5(1,2-5,0)$ & 0,009 & $16,0(6,1-41,0)$ & 0,000 \\
\hline 60 ou mais & $5,9(2,9-11,7)$ & 0,000 & $29,0(11,1-75,4)$ & 0,000 \\
\hline \multicolumn{5}{|l|}{ IMC * } \\
\hline Baixo peso/Normal & 1,0 & & 1,0 & \\
\hline Excesso de peso & $2,0(1,21-3,3)$ & 0,006 & $1,5(0,9-2,6)$ & 0,097 \\
\hline \multicolumn{5}{|l|}{ Margem } \\
\hline Direita & 1,0 & & 1,0 & \\
\hline Esquerda & $0,8(0,5-1,2)$ & 0,274 & $0,9(0,6-1,5)$ & 0,836 \\
\hline \multicolumn{5}{|l|}{ Localização } \\
\hline Jusante & 1,0 & & 1,0 & \\
\hline Montante & $0,4(0,3-0,7)$ & 0,000 & $0,5(0,3-0,8)$ & 0,007 \\
\hline Reserva de Cuniã & $0,4(0,2-0,8)$ & 0,006 & $0,7(0,3-1,3)$ & 0,224 \\
\hline \multicolumn{5}{|l|}{ Tabagismo } \\
\hline Não & 1,0 & & 1,0 & \\
\hline Sim & $0,9(0,6-1,4)$ & 0,768 & $1,6(1,0-2,6)$ & 0,035 \\
\hline \multicolumn{5}{|c|}{ Etilismo (vezes por semana) } \\
\hline Nenhuma & 1,0 & & 1,0 & \\
\hline 1 ou mais & $1,1(0,7-1,7)$ & 0,519 & $0,6(03-1,0)$ & 0,073 \\
\hline \multicolumn{5}{|c|}{ Consumo de peixe (vezes por semana) } \\
\hline Mais de 3 & 1,0 & & 1,0 & \\
\hline Até 3 & $1,3(0,8-2,0)$ & 0,200 & $0,8(0,5-1,3)$ & 0,404 \\
\hline \multicolumn{5}{|l|}{ Triglicerídeos ** } \\
\hline Normal & 1,0 & & 1,0 & \\
\hline Elevado & $1,5(0,8-2,9)$ & 0,235 & $3,9(2,1-7,1)$ & 0,000 \\
\hline \multicolumn{5}{|l|}{ 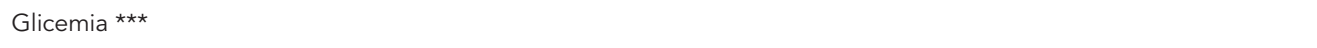 } \\
\hline Normal & 1,0 & & 1,0 & \\
\hline Elevada & $1,8(1,1-3,4)$ & 0,045 & $4,1(2,2-8,0)$ & 0,000 \\
\hline \multicolumn{5}{|c|}{ Escolaridade (anos de estudo) } \\
\hline Mais de 10 & 1,0 & & 1,0 & \\
\hline $3-9$ & $1,6(0,8-3,2)$ & 0,139 & $1,4(0,7-2,7)$ & 0,267 \\
\hline Menos de 2 & $2,5(1,1-5,7)$ & 0,029 & $3,8(1,7-8,1)$ & 0,000 \\
\hline \multicolumn{5}{|c|}{ Renda (salários mínimos) \# } \\
\hline Mais de 3 & 1,0 & & 1,0 & \\
\hline $1-3$ & $1,6(0,9-3,0)$ & 0,086 & $1,7(0,9-3,0)$ & 0,087 \\
\hline Menos de 1 & $1,5(0,8-2,7)$ & 0,210 & $1,6(0,8-3,0)$ & 0,145 \\
\hline
\end{tabular}

IC95\%: intervalo de 95\% de confiança; IMC: índice de massa corporal.

* Baixo peso/normal: IMC < 25kg.m-2; excesso de peso: IMC $\geq 25 \mathrm{~kg} \cdot \mathrm{m}^{-2}$;

** Normal: triglicerídeos $<150 \mathrm{mg} \%$; elevado: triglicerídeos $\geq 150 \mathrm{mg} \%$;

*** Normal: glicemia < $100 \mathrm{mg} \%$; elevada: glicemia $\geq 100 \mathrm{mg} \%$;

\# Valor de referência para um salário mínimo: $\mathrm{R} \$ 512,00$. 
Odds Ratio ajustada $\left(\mathrm{OR}_{\text {ajustada }}\right)$ para hipertensão arterial sistêmica (HAS), estratificada por sexo de acordo com características sociodemográficas, hábitos de vida e local de residência da população ribeirinha. Porto Velho, Rondônia, Brasil (2009-2011).

\begin{tabular}{|c|c|c|c|c|}
\hline & $\begin{array}{c}\text { Masculino } \\
\text { OR }_{\text {ajustada }}(\text { IC95\%) }\end{array}$ & Valor de $p$ & $\begin{array}{c}\text { Feminino } \\
\text { OR }_{\text {ajustada }}(\text { IC95\%) }\end{array}$ & Valor de $p$ \\
\hline \multicolumn{5}{|l|}{ Idade (anos) } \\
\hline $16-29$ & 1,0 & & 1,0 & \\
\hline $30-39$ & $0,7(0,3-1,8)$ & 0,567 & $6,4(1,8-24,0)$ & 0,005 \\
\hline $40-49$ & $1,0(0,4-2,5)$ & 0,901 & $7,6(2,0-29,0)$ & 0,003 \\
\hline $50-59$ & $2,1(0,93-4,9)$ & 0,071 & $15,3(3,8-60,0)$ & 0,000 \\
\hline 60 ou mais & $5,2(2,3-12,0)$ & 0,000 & $47,0(11,1-201,1)$ & 0,000 \\
\hline \multicolumn{5}{|l|}{$\mathrm{IMC} *$} \\
\hline Baixo peso/Normal & 1,0 & & - & - \\
\hline Excesso de peso & $2,6(1,4-4,6)$ & 0,001 & - & - \\
\hline \multicolumn{5}{|l|}{ Localização } \\
\hline Jusante & 1,0 & & - & - \\
\hline Montante & $0,4(0,2-0,7)$ & 0,001 & - & - \\
\hline Reserva de Cuniã & $0,4(0,2-0,8)$ & 0,003 & - & - \\
\hline \multicolumn{5}{|l|}{ Triglicerídeos ** } \\
\hline Normal & - & - & 1,0 & \\
\hline Elevado & - & - & $2,14(1,1-4,2)$ & 0,029 \\
\hline \multicolumn{5}{|l|}{ 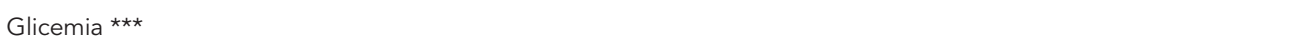 } \\
\hline Normal & 1,0 & & 1,0 & \\
\hline Elevada & $1,8(1,0-3,4)$ & 0,045 & $2,1(1,0-4,4)$ & 0,050 \\
\hline
\end{tabular}

IC95\%: intervalo de 95\% de confiança; IMC: índice de massa corporal.

* Baixo peso/normal: IMC < 25kg.m-2; excesso de peso: IMC $\geq 25 \mathrm{~kg} \cdot \mathrm{m}^{-2}$;

* Normal: triglicerídeos $<150 \mathrm{mg} \%$; elevado: triglicerídeos $\geq 150 \mathrm{mg} \%$;

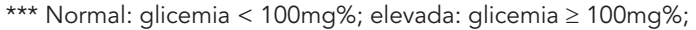

\section{Discussão}

Os projetos de desenvolvimento de infraestrutura na Amazônia Brasileira têm agregado uma série de questões geradoras de impactos socioambientais tais como migração acelerada, aumento da frota de veículos e maior demanda por educação e serviços de saúde nas redes pública e privada. Desde 2009, Porto Velho vem se destacando em cenário nacional por apresentar um complexo e intenso processo de desenvolvimento em função da construção das usinas hidrelétricas de Jirau e de Santo Antônio. Estudos mostram que investimentos como esses podem interferir na dinâmica social, ambiental e econômica local e alterar as condições de vida das populações tradicionais, como as comunidades ribeirinhas 12,13,14. Portanto, essas transformações influenciam diretamente na complexa rede de fatores que condicionam a saúde desses grupos populacionais 13 .

Fearnside 14 relatou potenciais alterações da qualidade de vida, mudanças nos hábitos alimentares, sociais e culturais, assim como a introdução de novos fatores de risco em grupos populacionais que residem próximos aos locais de construção de usinas hidrelétricas na Amazônia. A introdução de uma gama de riscos socioambientais poderá futuramente acarretar mudanças relevantes no perfil de morbimortalidade no que se refere, por exemplo, a doenças cardiovasculares 13 . As alterações ambientais decorrentes da construção da Usina Hidrelétrica Santo Antônio podem, por exemplo, influenciar o hábito alimentar das populações ribeirinhas e, consequentemente, aumentar a prevalência de HAS.

Apesar de uma afecção comum, de fácil diagnóstico e tratamento, a HAS está entre as causas modificáveis de mortalidade cardiovascular precoce mais importante em todo mundo, sobretudo, tendo em conta os acidentes vasculares encefálicos 7,15. No Brasil, cerca de $90 \%$ da população acometida por acidente vascular cerebral e $40 \%$ das vítimas de infarto agudo do miocárdio apresentaram como condição associada à HAS 16 . 
Os resultados do presente estudo mostraram que a prevalência de HAS nessas comunidades ribeirinhas foram superiores à prevalência encontrada em outros estudos nacionais realizados em áreas da Amazônia Brasileira. Estudos sobre a prevalência de HAS e fatores associados a essa condição foram realizados na cidade de Sinop, localizada ao norte do Estado de Mato Grosso, e em Belém, capital do Estado do Pará. Sinop apresentou prevalência de $23 \%$ 9; em Belém, a prevalência foi de $17 \%$ 17. Em nosso estudo, a prevalência de HAS foi de $25 \%$ para as regiões ribeirinhas de Porto Velho.

No que respeitante à diferença entre os sexos, a prevalência de HAS foi maior entre os homens, contrapondo alguns estudos nacionais e corroborando estimativas de estudos internacionais 15,18,19. Em países como Estados Unidos e Canadá, a prevalência de HAS foi estimada em 2003 em torno de 30\% para homens e $25 \%$ para mulheres. Na Europa, esses valores variaram entre $44,8 \%$ e $60,2 \%$ nos homens e $30,6 \%$ a $50,3 \%$ nas mulheres 20 . Ainda que as mulheres sejam apontadas como grupo de maior prevalência para HAS, um recente estudo mostrou uma tendência anual crescente dessa condição entre os homens 21 .

O risco de complicações de HAS também difere entre sexo, sendo maior entre homens quando comparado às mulheres durante a idade adulta; os homens tendem a desenvolver doenças cardiovasculares com maior frequência 22 . Em contraposição, as mulheres são mais preocupadas com estado de saúde e procuram mais assistência médica e, portanto, podem apresentar detecção precoce e maior controle da HAS quando comparadas aos homens 23 . Em nosso estudo, os fatores preditores para HAS foram distintos entre homens e mulheres. Para homens, o local de residência e o excesso de peso foram significantemente associados à maior prevalência de HAS. Entre as mulheres, além da idade, os triglicerídeos elevados estiveram relacionados à maior prevalência de HAS. A glicemia elevada esteve associada com HAS tanto em mulheres quanto nos homens. O excesso de peso já foi reportado como fator de risco associado à prevalência de HAS em outros trabalhos 23,24.

Em relação à idade, os resultados encontrados são semelhantes àqueles apontados na literatura nacional e internacional, em que a HAS aumenta em relação às categorias de idade $15,25,26,27$. No entanto, a prevalência de HAS na região ribeirinha difere entre as faixas etárias de algumas localidades do Brasil. Entre os indivíduos na faixa etária de 16-29 anos, a prevalência de HAS foi maior quando comparada à prevalência de estudos realizados em Sinop 9,
Salvador (Bahia) 15, Pelotas (Rio Grande do Sul) 18 e Lages (Santa Catarina) 19. Em relação à faixa etária e ao sexo, os resultados encontrados mostraram que tanto para homens quanto para mulheres, a população ribeirinha apresenta menor prevalência de HAS em idades mais avançadas quando comparadas aos resultados encontrados em um estudo realizado em Salvador 15. Tais resultados podem ser explicados, por exemplo, pelos hábitos alimentares da população ribeirinha, que é baseada no consumo de peixe.

De fato, o menor consumo de peixe esteve associado ao aumento da prevalência de HAS em adultos do sexo masculino, embora esses resultados não tenham sido estatisticamente significantes. Sabe-se que o peixe é uma importante fonte proteica, rico em aminoácidos sulfurados e em ácidos graxos insaturados, o que o torna um importante fator protetor contra doenças cardiovasculares 28 e outros processos degenerativos associados a substâncias tóxicas oxidantes presentes no ambiente 29,30.

Quanto à escolaridade, a prevalência de HAS foi maior em indivíduos com menos de 2 anos de estudos. Esses resultados concordam com aqueles obtidos por outros estudos nacionais $9,17,18,19,27$. Ainda que neste estudo a renda familiar não tenha sido um fator de risco estatisticamente significativo, estudos anteriores demonstraram que quanto menor a renda familiar, maior a prevalência de HAS 8,18.

A maior prevalência de HAS em adultos residentes na seção à jusante do local de construção da Usina Hidrelétrica Santo Antônio mostrou que o local de residência constitui-se um fator importante para estimar a prevalência de HAS. Essa variável foi um fator preditivo para ocorrência de HAS nos homens, mesmo após o ajuste de outras variáveis. Esses resultados podem ser explicados pelas características socioambientais, estilo de vida e hábitos alimentares que são diferentes entre as comunidades. A Reserva de Cuniã, por exemplo, possui menor prevalência de HAS quando comparada às comunidades localizadas imediatamente à jusante do local da construção da Usina Hidrelétrica de Santo Antônio (relativamente menos isoladas).

A comunidade da Reserva de Cuniã reside em meio de uma rica biodiversidade, caracterizada por árvores frutíferas, plantas medicinais e vários grupos de animais silvestres e sua alimentação é baseada quase que exclusivamente no consumo de peixe. Cerca de $80 \%$ da população adulta dessa região consome peixe mais de 3 vezes na semana e tem acesso relativamente limitado ao açúcar e a outros itens com maior poder calórico. Essa característica pode estar influenciando diretamente na prevalência de HAS, particularmente 
em razão das propriedades nutritivas do consumo de peixe 29,30 .

Como se trata de um estudo descritivoobservacional, pode apresentar limitações tais como possibilidade de viés de seleção, perdas diferencias, envolvimento de fatores de confusão e achados aleatórios nos resultados. Contudo, a realização de um censo demográfico e inclusão de uma gama de fatores de confusão por intermédio do inquérito epidemiológico reduziram a possibilidade da ocorrência de vieses neste estudo. Outra limitação se refere às duas aferições da pressão arterial, que foram realizadas em um curto intervalo de tempo. Entretanto, tal estratégia foi necessária devido ao difícil acesso às comunidades.

Em relação às perdas durante o estudo, o grupo de participantes submetido à avaliação antropométrica não apresentou diferenças significativas em relação ao sexo (valor de $p=0,310$ ) e à faixa etária (valor de $\mathrm{p}=0,178$ ), quando comparados àqueles que não fizeram a avaliação. Resultados semelhantes foram encontrados para os indivíduos que realizaram exames laboratoriais tanto para sexo (valor de $\mathrm{p}=0,705$ ) quanto para faixa etária (valor de $\mathrm{p}=0,223$ ), sendo a maior diferença percentual notada em indivíduos na faixa etária de 30-39 anos. Esses resultados sugerem que não há razão para acreditar que as características demográficas tenham distorcido os resultados em qualquer direção.

A iniciativa do estudo na fase de pré-enchimento do reservatório foi fundamental para o conhecimento da situação de saúde dessas comunidades e seu posterior monitoramento e vigilância. A descrição da prevalência de HAS das populações ribeirinhas poderá contribuir para formulação de estratégias de saúde por parte da secretaria de saúde local, considerando as potenciais mudanças que poderão ocorrer como consequência do impacto do desenvolvimento econômico da região. Além disso, pesquisas de prevalência da HAS devem priorizar as áreas do país onde essa informação ainda não está disponível, incluindo outros fatores de risco para doenças cardiovasculares.

\section{Resumen}

Estimar la prevalencia de hipertensión entre los adultos que viven en las comunidades que bordean el río Madeira, antes de la puesta en marcha de la central hidroeléctrica de Santo Antônio. Estudio transversal sobre la prevalencia de la hipertensión llevado a cabo con 841 adultos. Se evaluó la información sobre las condiciones sociodemográficas, morbilidad, estilo de vida, consumo de pescado y los datos antropométricos. El método de regresión logística para estimar el odds ratio y sus intervalos de confianza. Entre los habitantes locales, el $26 \%$ (95\%CI: 23-29\%) de los adultos tenían hipertensión:
29\% entre los hombres (95\%CI: 24-33\%) y el 23\% en las mujeres (95\% CI: 19-27\% ). En los hombres, los factores asociados con la hipertensión fueron: edad, índice de masa corporal, glucosa en sangre, y el lugar de residencia. Entre las mujeres, los factores de riesgo son la edad, triglicéridos y glucosa. Los resultados de este estudio pueden contribuir al desarrollo de estrategias de supervisión para la prevención de eventos cardiovasculares por los órganos de salud.

Hipertensión; Ecosistema Amazónico; Prevalencia 


\section{Colaboradores}

B. F. A. Oliveira contribuiu com a análise de dados e com a elaboração do manuscrito. G. Abbad, C. F. Mariani, M. F. Fonseca e W. Bastos colaboraram na revisão e correção do manuscrito. A. V. Souza, J. M. C. Costa, N. Gomes, D. S. Mourão contribuíram com a construção do banco de dados, análises estatísticas e elaboração do manuscrito. S. Hacon trabalhou na elaboração e correção do manuscrito.

\section{Agradecimentos}

O projeto foi conduzido em conjunto com as instituições Escola Nacional de Saúde Pública Sergio Arouca e Instituto Nacional da Saúde da Mulher da Criança e do Adolescente Fernandes Figueira/Fundação Oswaldo Cruz, Universidade Federal de Rondônia, Universidade Federal do Rio de Janeiro, Pontifícia Universidade Católica e financiado pelo INOVA ENSP e Santo Antônio Energia. Agradecemos também a todos os participantes e ao apoio da Secretaria Municipal de Saúde de Porto Velho.

\section{Referências}

1. World Health Organization. Global status report no nocommunicable diseases 2010. http://www. who.int/nmh/publications/ncd_report2010/en/ (acessado em 28/Dez/2011).

2. Kearney PM, Whelton M, Reynolds K, Muntner P, Whelton PK, He J. Global burden of hypertension: analysis of worldwide data. Lancet 2005; 365 : 217-23.

3. Passos VMA, Assis TD, Barreto SM. Hipertensão arterial no Brasil: estimativa de prevalência a partir de estudos de base populacional. Epidemiol Serv Saúde 2006; 15:35-45.

4. Departamento de Análise de Situação de Saúde, Secretaria de Vigilância em Saúde, Ministério da Saúde. Vigilância de Fatores de Risco e Proteção para Doenças Crônicas por Inquérito Telefônico: VIGITEL 2009. Brasília: Ministério da Saúde; 2010.

5. Organização Pan-Americana da Saúde. Doenças crônico-degenerativas e obesidade: estratégia mundial sobre alimentação saudável, atividade física e saúde. Brasília: Organização Pan-Americana da Saúde; 2003.

6. Popkin BM. Global nutrition dynamics: the world is shifting rapidly toward a diet with noncommunicable disease. Am J Clin Nutr 2006; 84:289-98.

7. Sociedade Brasileira de Cardiologia. V diretrizes brasileiras de hipertensão arterial. Arq Bras Cardiol 2007; 89:e24-79.

8. Barbosa LS, Scala LCN, Ferreira MG. Associação entre marcadores antropométricos de adiposidade corporal e hipertensão arterial na população adulta de Cuiabá, Mato Grosso. Rev Bras Epidemiol 2009; 12:237-47.
9. Martins MSA, Ferreira MG, Guimarães LV, Vianna LAC. Hipertensão arterial e estilo de vida em Sinop, Município da Amazônia Legal. Arq Bras Cardiol 2010; 94:639-44.

10. Brandão AA, Magalhães MEC, Ávila A, Tavares A, Machado CA, Campana EMG, et al. Conceituação, epidemiologia e prevenção primária. J Bras Nefrol 2010, 32 Suppl 1:1-4.

11. Fagundes AA, Coitinho D. Vigilância alimentar e nutricional - SISVAN: orientações básicas para a coleta, processamento, análise de dados e informação em serviços de saúde. Brasília: Ministério da Saúde; 2004. (Série A. Normas e Manuais Técnicos).

12. Oliveira FAZ, Heukelbach J, Gomide M, Moura RCS. Grandes represas e seus impactos em saúde pública: II. Efeitos à jusante. Cad Saúde Colet (Rio J.) $2007 ; 15: 9-26$.

13. Rigotto RM. Inserção da saúde nos estudos de impacto ambiental: o caso de uma termelétrica a carvão mineral no Ceará. Ciênc Saúde Coletiva 2009; 14:2049-59.

14. Fearnside P. Hidrelétricas amazônicas e a política energética - Parte 1. http://colunas.globoama zonia.com/philipfearnside/ (acessado em 10/ Mar/2012).

15. Lessa I, Magalhães L, Araújo MJ, Almeida FN, Aquino E, Oliveira MMC. Hipertensão arterial na população adulta de Salvador (BA). Arq Bras Cardiol 2006; 87:747-56.

16. Castro ME, Rolim MO, Mauricio TF. Prevenção da hipertensão e sua relação com o estilo de vida de trabalhadores. Acta Paul Enferm 2005; 18:184-9. 
17. Borges HP, Cruz NC, Moura EC. Associação entre hipertensão arterial e excesso de peso em adultos, Belém, Pará, 2005. Arq Bras Cardiol 2008; 91:110-8.

18. Costa JSD, Barcellos FC, Sclowitz ML, Sclowitz IKT, Castanheira M, Olinto MTA, et al. Hypertension prevalence and its associated risk factors in adults:a population-based study in Pelotas. Arq Bras Cardiol 2007; 88:59-65.

19. Longo GZ, Neves J, Luciano VM, Peres MA. Prevalência de níveis pressóricos elevados e fatores associados em adultos de Lages/SC. Arq Bras Cardiol 2009; 93:387-94.

20. Wolf-Maier K, Cooper RS, Banegas JR, Giampaoli $\mathrm{S}$, Hense HW, Joffres M, et al. Hypertension prevalence and blood pressure levels in 6 European countries, Canada and United States. JAMA 2003; 289:2363-9.

21. Muniz LC, Cascaes AM, Wehrmeister FC, MartínezMesa J, Barros AJD, Menezes AMB. Trends in selfreported arterial hypertension in Brazilian adults: an analysis of data from the Brazilian National Household Sample Survey, 1998-2008. Cad Saúde Pública 2012; 28:1599-607.

22. Noblat ACB, Lopes MB, Lopes GB, Lopes AA. Complicações da hipertensão arterial em homens e mulheres atendidos em um ambulatório de referência. Arq Bras Cardiol 2004; 83:308-13.

23. Saeed AA, Al-Hamdan NA, Bahnassy AA, Abdalla AM, Abbas MA, Abuzaid LZ. Prevalence, awareness, treatment, and control of hypertension among Saudi adult population: a national survey. Int J Hypertens 2011; 2011:174135.
24. Freitas OC, Carvalho FR, Neves JM, Veludo PK, Parreira RS, Gonçalves RM, et al. Prevalence of hypertension in the urban population of Catanduva, in the State of São Paulo, Brazil. Arq Bras Cardiol 2001; 77: 9-21.

25. Barreto SM, Passos VM, Firmo JO, Guerra HL, Vidigal PG, Lima-Costa MF. Hypertension and clustering of cardiovascular risk factors in a community in Southeast Brazil, The Bambui Health and Ageing Study. Arq Bras Cardiol 2001; 77:576-81.

26. Hartmann M, Dias-da-Costa JS, Olinto MTA, Pattussi MP, Tramontini A. Prevalência de hipertensão arterial sistêmica e fatores associados: um estudo de base populacional em mulheres no sul do Brasil. Cad Saúde Pública 2007; 23:1857-66.

27. Ferreira MG, Valente JG, Gonçalves-Silva RMV, Sichieri R. Consumo de bebida alcoólica e adiposidade abdominal em doadores de sangue. Rev Saúde Pública 2008; 42:1067-73.

28. Whelton SP, He J, Whelton PK, Muntner P. Metaanalysis of observational studies on fish intake and coronary heart disease. Am J Cardiol 2004; 93:1119-23.

29. Dórea JG, de Souza JR, Rodrigues P, Ferrari I, Barbosa AC. Hair mercury (signature of fish consumption) and cardiovascular risk in Munduruku and Kayabi Indians of Amazonia. Environ Res 2005; 97:209-19.

30. Houston MC. Role of mercury toxicity in hypertension, cardiovascular disease, and stroke. J Clin Hypertens 2011; 13:621-7.

Recebido em 08/Out/2012

Versão final reapresentada em 15/Fev/2013

Aprovado em 26/Mar/2013 